\title{
Less Invasive Surgery is Feasible in the Management of Traumatic Thoracolumbar Fractures in Isolated and Polytrauma Injury
}

\author{
I. SANLI, MD, ${ }^{1}$ A. SPOOR, MD ${ }^{2}$ S.P.J. MUIJS, MD ${ }^{3}$ F.C. ÖNER, $\mathrm{MD}^{3}$ \\ ${ }^{I}$ Department of Orthopaedic Surgery, Maastricht University Medical Centre+, the Netherlands, ${ }^{2}$ Department of Orthopaedic Surgery, Elisabeth Hospital Tilburg, \\ the Netherlands, ${ }^{3}$ Department of Orthopaedic Surgery, Utrecht University Medical Centre, the Netherlands
}

\begin{abstract}
Background: Less invasive stabilization systems (LISSs) have gained popularity. However, limited quality of life (QOL) and clinical outcome data exist for trauma patients treated with LISSs. The objective of this study is to describe QOL and outcome for posterior percutaneous pedicle screw fixation in the management of traumatic thoracolumbar fractures.

Methods: Between January 2006 and December 2011, data from all patients treated with a posterior percutaneous pedicle screw fixation technique for thoracolumbar fractures were collected and analyzed. Sixty-nine patients met the inclusion criteria. Additional vertebral reduction and cement augmentation was used in 25 patients, when there was more than $50 \%$ of vertebral body comminution.

Results: Mean follow up of 19 months (range $=6-49$ months). Fifty-one percent of the study population consisted of polytrauma patients, with $22 \%$ having injury severity score $\geq 15$. In 6 cases $(8.7 \%)$ there were perioperative complications. Response rate for the follow-up health survey was $78 \%$, with a satisfactory overall median EuroQuol score of 0.811 (Q1-Q3 95\% confidence interval = 0.709-0.897).

Conclusions: Posterior percutaneous pedicle screw fixation proves to be effective in the management of traumatic thoracolumbar fractures, with a good overall functional outcome. Percutaneous techniques that reduce perioperative morbidity are an alternative approach well suited for damage control orthopaedics, as long as there are no neurological deficits. Especially in polytrauma patients with spine fractures, the spinal column can be stabilized in an emergency setting, while limiting the risks of "a second hit" at the patients' already frail condition.
\end{abstract}

Level of Evidence: 3.

Minimally Invasive Surgery

Keywords: less invasive surgery, pedicle screw fixation, thoracolumbar fracture, quality of life

\section{INTRODUCTION}

The management of traumatic thoracolumbar fractures remains challenging. The levels of evidence for treatment practices can alter the decisionmaking process. In the new era, percutaneous pedicle screw fixation has become a popular method as a less invasive approach in the management of thoracolumbar fractures. Several studies have described the multiple advantages of posterior pedicle screw fixation techniques in thoracolumbar fractures. $^{1-9}$

Open surgical techniques are associated with significant morbidity due to high blood loss and infection rates. Blood loss rates of $1000 \mathrm{~mL}$ for open posterior, anterior, or anterior-posterior procedures can be reduced to $50 \mathrm{~mL}$ in less invasive spine surgery. The high infection rate of $10 \%$ in open surgery is reduced to $0-1 \% .^{4-9}$ In contrast to open techniques, percutaneous fixation induces minimal paraspinal muscle injury and shows a positive correlation with postoperative back muscle performance. $^{10}$

With the knowledge that, on average, $36 \%$ of polytrauma patients have associated spine injuries, less invasive approaches would be favorable in limiting the risks for the already vulnerable patient. ${ }^{11}$ However, it is not yet established whether less invasive approaches lead to comparable clinical outcome in the isolated and/or the multi-injured patient. To our knowledge, little is known about health-related quality of life (QOL) outcomes. The low-grade evidence and unclear long-term outcomes further limit the evidence available for this tech- 
nique. There is a need for more evidence to inform clinical decisions using percutaneous pedicle screw fixation in the treatment of traumatic thoracolumbar fractures.

The aim of this study is to describe the QOL and radiological outcome of posterior percutaneous pedicle screw fixation in the treatment of traumatic thoracolumbar fractures from a single trauma center.

\section{MATERIAL AND METHODS}

Our center started using the posterior percutaneous pedicle screw fixation technique for traumatic thoracolumbar spine fractures in January 2006. All trauma patients in our center are included in a prospective registry. The primary outcome of our study was to analyze the functional and radiological outcome of patients from this database. For this purpose, all patients with traumatic thoracolumbar fractures treated with a percutaneous spinal fixation technique between January 2006 and December 2011 were included. Follow-up data were extracted from electronic medical records. Excluded were all patients with a follow up less than 6 months after surgery, pathological vertebral body fractures, or accompanying neurological symptoms necessitating open decompression. Classification type $\mathrm{C}$ was excluded from EuroQuol (EQ-5D) analysis because only a single observation was available. Seventeen patients were lost to follow up (relocation, no-show) during this period; a total of 69 patients remained for analysis.

Spine stabilization was performed by use of the percutaneous multilevel implant fixation system $\mathrm{CD}$ Horizon $^{\circledR}$ Longitude (Medtronic, Memphis, TN, USA). In 3 patients, the SpiRIT ${ }^{\circledR}$ system (Synthes $\mathrm{GmbH}$, Oberdorf, Switzerland) was used. In 46 patients, short-segment pedicle screw instrumentation with bilateral pedicle screws (1 level above and 1 below the fracture) was performed. In 23 patients, a long-segment fixation ( 2 or more levels above and below fracture) was performed, with 7 patients having fractures at 2 or more levels. Additional vertebral balloon assisted endplate reduction (BAER) and cement augmentation techniques were used when substantial vertebral body comminution (more than $50 \%$ ) was seen on the preoperative computed tomography (CT) scans. In 25 patients with substantial comminution of the vertebral body, this combined technique with percutaneous anterior column augmentation was performed. During the
Table 1. Baseline patient characteristics.

\begin{tabular}{lc}
\hline Baseline Characteristics & Value, N $=\mathbf{6 9}$ \\
\hline Gender, No. (\%) & $48(70)$ \\
Male & $21(30)$ \\
Female & $45.58( \pm 16.59)$ \\
Age (y), mean (SD) & $5(7)$ \\
Underlying osseous pathology, No. (\%) & $2(3)$ \\
Osteoporosis & $35(51)$ \\
Ankylosing spondylitis & $15(22)$ \\
Polytrauma patients, No. (\%) & $20(29)$ \\
ISS $\geq 15$ & \\
ISS < 15 & $41(55)$ \\
AOSpine classification, No. fractures (\%) & $4(5)$ \\
Type A (0,1,2,3,4) & $37(50)$ \\
Type A 0-2 & $33(44)$ \\
Type A 3-4 & $1(1)$ \\
Type B (1,2,3) & $17(25)$ \\
Type C & $37(54)$ \\
Fracture location, No. (\%) & $15(22)$ \\
Thoracic & \\
Thoracolumbar & $46(67)$ \\
Lumbar & $23(33)$ \\
Segment fixation, No. (\%) & \\
Short segment & \\
Long segment & \\
\hline Abbrevations: & \\
\hline
\end{tabular}

Abbreviations: ISS, injury severity score; SD, standard deviation.

initial phase of inclusion, some of the surgeons in our group decided to use bracing following operative spinal stabilization (Table 1). All fractures were classified according to the AOSpine classification (Figure 1). ${ }^{12}$

QOL was determined by use of EQ-5D. A survey was sent to all patients after the last follow-up visit. The EQ-5D is an instrument designed to measure generic health status across 5 dimensions of health: mobility, self-care, usual activities, pain/discomfort, and anxiety/depression, with 3 response levels (no problems, some problems, extreme problems). ${ }^{13} \mathrm{~A}$ unique EQ-5D health state is defined by combining 1 level from each of the 5 dimensions, and scores range from -0.109 to 1.0 , with score 1 indicating the best overall health. For validation of our EQ-5D results, we used the time tradeoff for the Dutch population. ${ }^{14}$

The radiological outcome was assessed (and its reliability tested) by 2 reviewers (I.S. and A.S.), measuring the local kyphosis and segmental wedge angles on supine preoperative and standing postoperative and follow-up x-rays. Kyphosis angle was defined as the measured angle between the superior and inferior endplates of the fractured vertebra and segmental angle as the angle of the stabilized segments measured between the superior endplate of the upper instrumented vertebra and the inferior endplate of the lower instrumented vertebra. 


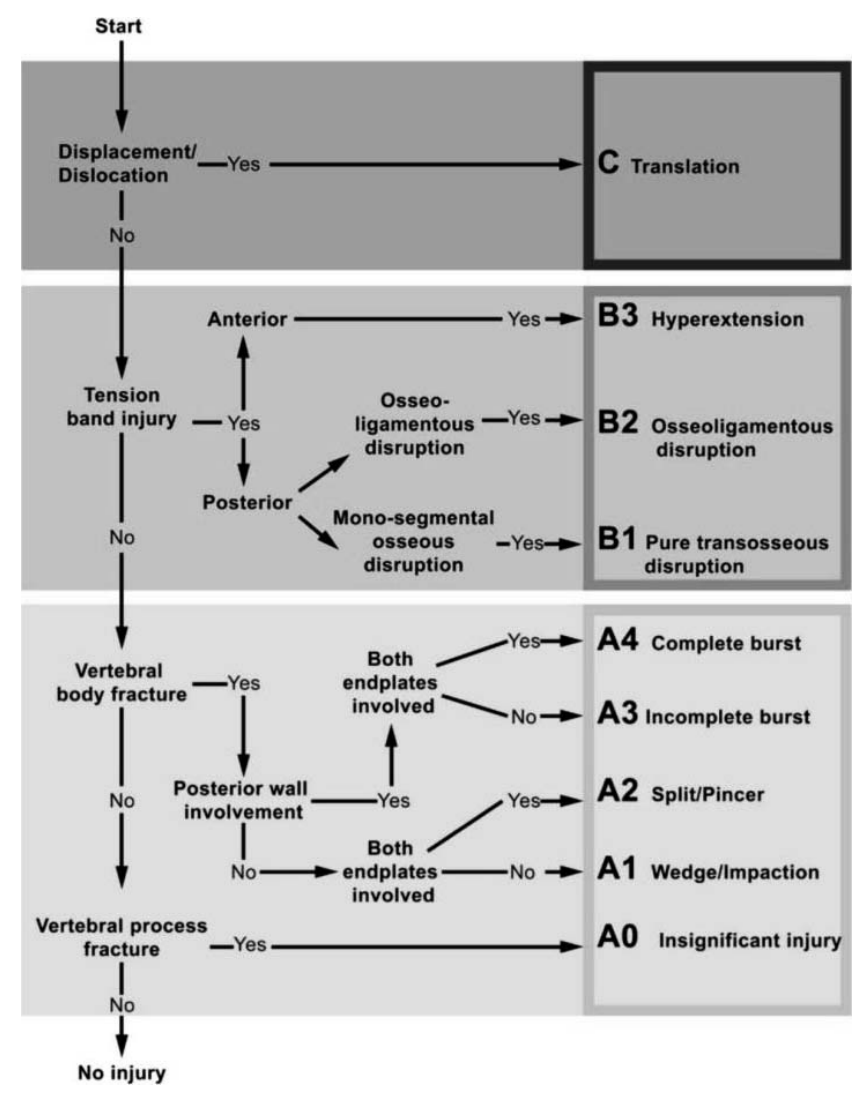

Figure 1. AOSpine classification.

\section{Statistical Methods}

Analyses were done using the $\mathrm{R}$ statistical package version 15.2 , the car and irr packages. ${ }^{15,16}$ Baseline variables are given as absolute number and percentages when describing categorical data. For continuous variables, the mean with standard deviation (SD) is given. If the observations were nonnormally distributed, the median (Q2) and first and third quartiles (Q1-Q3) are given. The average agreement between reviewers determining the kyphosis and segmental outcomes was evaluated using the intraclass correlation coefficient (ICC) at follow up. Q-Q plots were used to determine if the outcome residuals were normally distributed. To evaluate surgery effectiveness, the kyphotic or segmental angles, postsurgery and during follow-up, were subtracted from the presurgery angles. Changes over time were analyzed using a mixed model with a random intercept for patient, time of measurement, and adjustment for baseline (presurgery) angle. As a sensitivity analysis, we corrected for differences in follow-up time, showing no improved fit. Approximate $95 \%$ confidence intervals (CIs) were calculated based on the pooled standard error and a $t$ distribution with 68 degrees of freedom. Finally, given that the residuals of the EQ-5D were not normally distributed, which could not be ameliorated by customary transformation, Wilcoxon tests, with a continuity correction, were used.

\section{RESULTS}

\section{Clinical Outcome and EQ-5D}

Within the cohort of 86 patients, 69 patients fulfilled the inclusion criteria for the study. The baseline characteristics of the study are described in Table 1. The mean follow up was 19 months (range $=6-49$ months). The study population consisted of a high percentage of men, with a high overall percentage of polytrauma patients in an average young study population, with a mean age of 46 years. The median blood loss for subjects with short-segment fixation was $50 \mathrm{~mL}$ (range $=10-100$ $\mathrm{mL}$ ) and for long-segment fixation $108 \mathrm{~mL}$ (range $=50-500 \mathrm{~mL} ; P=0.05$ ).

The response rate for the follow-up health survey was $78 \%$, with an overall median EQ-5D score of 0.811 (Q1-Q3 95\% CI $=0.709-0.897 ; \quad P<0.01$ ). EQ-5D scores were nonsignificantly different, for subjects younger than 50 years of age $(\mathrm{Q} 2=0.843)$ and older $(\mathrm{Q} 2=0.811 ; P=0.57)$. Stratifying EQ-5D for males and females again did not show significant difference in distributions $(P=0.72)$. EQ-5D did not differ significantly for subjects with polytrauma $(\mathrm{Q} 2=0.811)$ versus no polytrauma $(\mathrm{Q} 2=0.843$; $P=0.46)$. The median EQ-5D scores for AOSpine classification type A and B were 0.827 and 0.811 . The Spearman correlation of AOSpine classification type $\mathrm{A}$ and $\mathrm{B}$ to EQ-5D was -0.13 , suggesting that the EQ-5D decreases with classification type B; however, these associations were not significant. Patients who underwent a long-segment fixation showed no significant difference in EQ-5D $(\mathrm{Q} 2=0.827$ versus 0.811 in short-segment fixation; $P=0.84$ ).

\section{Complications}

In $8.7 \%$ (6 cases), we found perioperative complications. In 4 patients, possible cerebrospinal fluid leakage was observed during insertion of the Jamshidi needles in the pedicles. After repositioning, there were no further consequences. In 1 patient, ventral K-wire migration was observed fluoroscopically during surgery without any consequences. In another patient, a loosening of the balloon was seen in a noninflated stent during a stenting procedure, without clinical consequences. In the postoperative 
Table 2. Complications of spinal fixation.

\begin{tabular}{lc}
\hline Type of Complication & Number \\
\hline Preoperative & \\
Suspected dural lesion & 4 \\
K-wire migration & 1 \\
BAER balloon dislocation & 1 \\
Postoperative & 1 \\
Hematoma & 1 \\
Rod dislocation & 1 \\
Bend rod & 1 \\
Dislocation of set screw & 1 \\
Screw breakage & 2 \\
Lumbosacral plexopathy & 2 \\
Wound infection & 1 \\
Wound leakage & 1 \\
Subsidence in kyphosis & 5 \\
Pneumonia & 2 \\
Urinary tract infection & 1 \\
Atrial fibrillation & 1 \\
Ileus & 1 \\
Bladder retention & 1 \\
Respiratory insufficiency & 1 \\
Pneumothorax & \\
\hline
\end{tabular}

Abbrevation: BAER, balloon assisted endplate reduction.

period, cardiopulmonal, urogenital, and gastrointestinal complications predominated with a total postoperative risk of $30 \%$. The mortality rate of the whole group was $2.9 \%$, not procedure related. Polytrauma patients had an increased risk of perioperative complications as shown by the risk difference $(\mathrm{RD})$ of $0.03(95 \% \mathrm{CI}=-0.09-0.15)$ and postoperative complications $(0.25,95 \% \mathrm{CI}=0.04$ 0.46). The RD for perioperative complications due to long-segment fixation was $0.09(95 \% \mathrm{CI}=-0.06-$ 0.24 ), and for postoperative complications, the RD was $0.13(95 \% \mathrm{CI}=-11-0.37)$. There was a deep infection rate of $3 \%$ ( 2 cases). In both patients, the material was extracted 3 months postoperatively. There was a $6 \%$ material failure rate with 2 patients experiencing dislocations of material (Table 2).

\section{Radiological Outcome}

ICC at follow up between the 2 raters was 0.93 (95\% CI $=0.89-0.96)$ for kyphosis and $0.98(95 \%$ $\mathrm{CI}=0.97-0.99)$ for the segmental angles. Assuming no difference over time, this indicates that agreement between observers is overall very high and even higher for the segmental angles. Our study showed a kyphosis correction with a kyphotic angle mean of $3.26^{\circ}$ and segmental angle mean of $1.81^{\circ}(P<0.01)$.

Loss of correction was calculated by subtracting follow up from postoperative measurement. The mean kyphotic subsidence was $-1.99,-1.45$, and $-1.79^{\circ}$ for thoracic, thoracolumbar, and lumbar locations, respectively $(P=0.85)$. The mean segmental subsidence was $-5.11,-4.19$, and $-2.30^{\circ}$ for thoracic, thoraco-
Table 3. Primary outcome results.

\begin{tabular}{|c|c|c|}
\hline Outcomes & Point Estimates & $\begin{array}{l}\text { Mean Difference } \\
(95 \% \text { CI) } P \text { Value }\end{array}$ \\
\hline Wedge mean (SD) & & Reference overall \\
\hline Pre-operation & $13.35(6.74)$ & $P<0.01^{*}$ \\
\hline Postoperation & $10.09(5.54)$ & $3.26(2.06-4.45)$ \\
\hline Last follow up & $11.75(6.21)$ & $1.60(0.40-2.79)$ \\
\hline Segmental mean (SD) & & Reference overall \\
\hline Pre-operation & $17.45(11.34)$ & $P<0.01^{*}$ \\
\hline Postoperation & $15.64(11.83)$ & $1.81(0.05-3.56)$ \\
\hline Last follow up & $19.65(11.37)$ & $-2.20(-3.96$ to -0.45$)$ \\
\hline EQ-5D median (Q1-Q3)† & $0.811(0.709-0.897)$ & $P<0.01 \ddagger$ \\
\hline
\end{tabular}

Abbreviations: CI, confidence interval; EQ-5D, EuroQuol; SD, standard deviation.

*The overall $P$ values for the wedge and segmental outcomes are based on a mixed model with random intercept for patient and adjusted for baseline angle.

$\dagger$ First and third quartiles (Q1-Q3) are given.

\$Mann-Whitney test; null-hypothesis population EQ-5D is 0.450 .

lumbar, and lumbar locations, respectively $(P=0.32)$. The mean subsidence, based on the segmental angle was $-4.66^{\circ}$ for younger subjects $(<50$ years of age) and $-3.06^{\circ}$ for older subjects $(P=0.23)$. For the kyphotic angle, the mean was $-1.64^{\circ}$ for younger subjects and $-1.69^{\circ}$ for older subjects $(P=0.95)$. When we correlated an additional intervention (anterior column augmentation) to percutaneous pedicle screw fixation, the mean subsidence was $-4.43^{\circ}$ for the segmental angle in patients receiving kyphoplasty/vertebroplasty/vertebral body stenting (VBS) and $-3.77^{\circ}$ in subjects receiving percutaneous pedicle screw fixation as a standalone procedure $(P=0.65)$. For the kyphotic angle, the means were $-2.29^{\circ}$ in patients receiving BAER with augmentation interventions and $-1.30^{\circ}$ in subjects receiving no additional intervention $(P=0.32$; Table 3, Figure 2$)$.

\section{DISCUSSION}

At present, the evolution of less invasive stabilization systems (LISSs) is adding major goals to

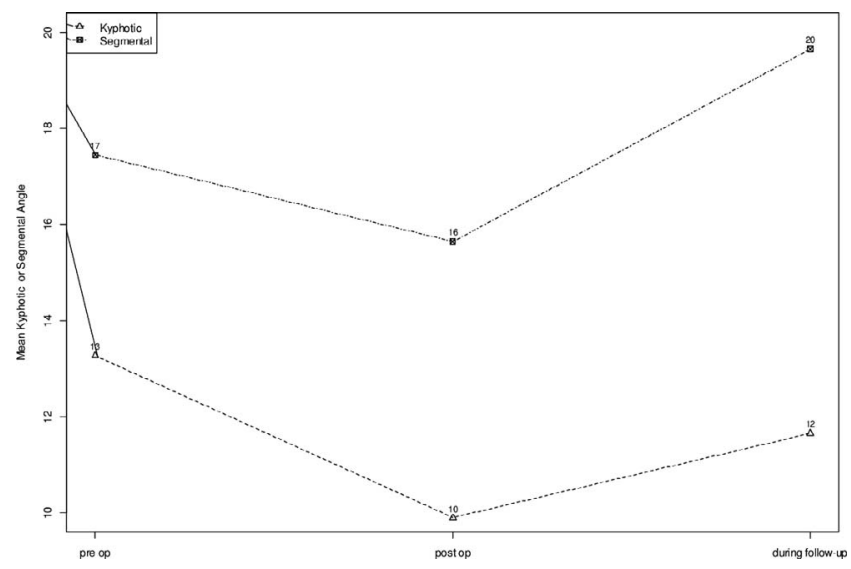

Figure 2. Mean wedge and segmental angles at presurgery, postsurgery, and at last follow up. 
spine surgery. Besides improving pain and neurologic deficit with a reduction of approach-related morbidity, spine surgery is focused on improving QOL. To date, several studies have described the multiple advantages of posterior pedicle screw fixation techniques in thoracolumbar fractures. ${ }^{1-9}$ However, QOL outcome data are limited for spine trauma patients. Besides that, the role of LISSs remains unclear in treating spine fractures in polytrauma patients.

Cimatti et al. evaluated Short-Form 36 questionnaire (SF-36) outcomes in a 2 year prospective study of percutaneous pedicle screw fixation in 32 patients with unstable single-level thoracolumbar fractures. Concerning the SF-36 physical scale, patients achieved 46.43 points for male, 46.19 for female patients, representing a better outcome than the back pain population (44.79) but worse compared with the scores achieved with the normal population (50.21). The average score achieved in the SF-36 psychological score was 56.22, which exceeds the scores from the back pain population (48.25) and the normal population (51.54). ${ }^{17}$ In the study of Schmidt et al., of the 76 patients with type A fractures who were treated with minimally invasive instrumentation, 32 patients $(42.1 \%)$ had no substantial discomfort and pain as compared before surgery. Six months following surgery, 58 patients $(76.3 \%)$ met their expectations or were highly pleased by their individual postoperative results. ${ }^{18}$ In our study, we used EQ-5D for evaluation of QOL and observed a significant high overall median EQ-5D score of 0.811 (Q1-Q3 95\% $\mathrm{CI}=0.709-0.897)$. The EQ-5D results of the nonspecific low back pain population is 0.731 $(\mathrm{SD}=0.172) .{ }^{19} \mathrm{We}$ found no statistical significant difference in EQ-5D outcome when EQ-5D was stratified for gender, age, polytrauma, AOSpine classification, or long-segment fixation.

Despite results in improvement of sagittal alignment and kyphosis correction, numerous studies report loss of correction during follow up for pedicle screw fixation. ${ }^{5-6,8}$ However, there is no clear correlation between the loss of correction and clinical results. Wild et al. describe a retrospective analysis of a fixation alone technique for type A fractures using a percutaneous internal fixator in 10 cases and an open procedure in 11 cases. ${ }^{5}$ Five years after implant removal, the loss of correction of the bisegmental wedge angle averages $7.62^{\circ}$ (median $7^{\circ}$; range $=0-20^{\circ} ; \mathrm{SD}=4.5^{\circ}$ ) in both groups; however, neither in the Hannover-Spine-Score nor in the SF36 Health Questionnaire did these groups show any difference. In our study, we found a mean subsidence of $1.60^{\circ}$ for the kyphotic angle and $2.20^{\circ}$ for the segmental wedge angle at the end of the followup period with a high QOL. Our use of augmentation techniques for substantial comminution has probably limited complications and loss of correction. In our study, significant comminution of the vertebral body resulted in the same radiological outcome as less comminuted fractures.

In the trauma population, which is prone for infection and blood loss, lower infection rates and minimal blood loss are described for percutaneous pedicle screw fixation compared to the open techniques. Infection rates of $3.1-10 \%$ for the open surgical technique described by Verlaan et al. can be reduced to $0-1 \%$ by use of the percutaneous pedicle screw. ${ }^{4}$ Minimal blood loss rates of $50 \mathrm{~mL}$ are described in percutaneous pedicle screw techniques. ${ }^{5-9}$ In our study, the infection rate and blood loss was comparable to other studies.

Posterior percutaneous pedicle screw fixation can be performed fast and less invasively in the trauma patient. The technique allows immediate stable fixation because the screws transverse all 3 columns. Anatomic or best possible alignment of the spinal column is obtained. Operation time is reduced to an average of 78 minutes. ${ }^{1-9}$ Especially in polytrauma patients with spinal fractures, the spinal column can be stabilized in an emergency setting, while limiting the risks of the patients' condition. In chest trauma, patients can be mobilized early, preventing respiratory complications. ${ }^{19}$ The features of percutaneous pedicle screw fixation make the approach suitable for a damage control protocol. The circumstances of the critical polytrauma patient make it complex to supply level 1 evidence. However, many observational studies show a significant difference between patients who were treated with a spinal damage control regimen compared to a delayed surgery group by means of mean length of operative time, length of hospital stay, number of ventilator dependent days, and several early complications, such as wound and pulmonary complications and pressure sores. ${ }^{11,20-22}$ In our study, 31 patients with a type $B$ fracture (ASIA grade E) and 1 patient with a type $\mathrm{C}$ fracture (ASIA grade $\mathrm{A}$ ), with traumatic complete paraplegia who was hemodynamically unstable to perform open surgery, could be successfully stabilized by use of a percutaneous approach. 
In our opinion, posterior percutaneous pedicle screw fixation technique can be used for all spinal fractures when there are no neurologic problems.

Limitations of this study are the retrospective analysis of data, limited number of patients, and the heterogeneity of our population, with a large group of type A fractures. This case series represents also the learning curve of the technique. The overall young study population can confound the QOL outcomes because of general favorable results due to age. It should be noticed that our study comprises a high population $(51 \%)$ of polytrauma patients, with $22 \%$ who sustained more severe injury (injury severity score $[\mathrm{ISS}] \geq 15$ ). In our opinion, the high overall postoperative complication rate of $30 \%$ as shown in our study can be clarified with this high percentage of polytrauma patients. Another limitation of this study is the use of a standard time period for measurement for QOL outcome at the end of the follow-up period. To date, a disease-specific QOL score does not exist for spinal trauma patients, and there is no valid tool to obtain preinjury QOL data. Another shortcoming of the study is the sparse use of CT scans postoperatively for fusion assessment. We only used CT scans postoperatively when patients presented with complaints. In our opinion, use of routine standard x-rays in combination with clinical results are adequate and satisfactory to assess alignment, material failure, and fracture healing. The relatively long follow-up period with a mean of 19 months (range $=6-49$ months) comprising all spinal fracture locations including AO type B fractures and standardized outcome assessment (EQ-5D) represent the strength of this article.

\section{CONCLUSIONS}

Percutaneous pedicle screw fixation can be recommended in the management of traumatic thoracolumbar fractures, as well as in polytrauma cases when decompressive surgery is not necessary. Development of percutaneous spine approaches that reduce perioperative morbidity can be a good alternative approach following the damage control principles. The technique has a good overall functional outcome.

Given the heterogeneity and the lack of robust evidence, these findings warrant verification in larger prospective registries and randomized controlled trials.

\section{REFERENCES}

1. Wang H, Zhou Yue, Li C, et al. Comparison of open versus percutaneous pedicle screw fixation using the sextant system in the treatment of traumatic thoracolumbar fractures. Clin Spine Surg. 2017;30(3):E239-246. doi: 10.1097/BSD. 0000000000000135.

2. Barbagallo GMV, Raudino G, Visocchi $M$, et al. Restoration of thoracolumbar spine stability and alignment in elderly patients using minimally invasive spine surgery (MISS). A safe and feasible option in degenerative and traumatic spine diseases. Acta Neurochirurg Suppl. 2017;124:69-74. doi: 10. 1007/978-3-319-39546-3_11.

3. Korovessis P, Mpountogianni E, Vasilleios S. Percutaneous pedicle screw fixation plus kyphoplasty for thoracolumbar fractures A2, A3 and B2. Eur Spine J. 2017;26(5):1492-1498. doi: 10.1007/s00586-016-4743-6.

4. Verlaan JJ, Dhert WAJ, Verbout AJ, et al. Balloon vertebroplasty in combination with pedicle screw instrumentation: a novel technique to treat thoracic and lumbar burst fractures. Spine. 2005;30(3):73-79.

5. Wild MH, Glees M, Plieschnegger C, et al. Five-year follow-up examination after purely minimally invasive posterior stabilization of thoracolumbar fractures: a comparison of minimally invasive percutaneously and conventionally open treated patients. Arch Orthop Trauma Surg. 2007;127(5):335-343.

6. Pelegri C, Benchikh El Fegoun A, Winter M, et al. Percutaneous osteosynthesis of lumbar and thoracolumbar spine fractures without neurological deficit: surgical technique and preliminary results. Rev Chir Orthop Reparatrice Appar Mot. 2008;94(5):456-463. doi: 10.1016/j.rco.2008.03.035.

7. Merom L, Raz N, Hamud C, et al. Minimally invasive burst fracture fixation in the thoracolumbar region. Orthopedics. 2009;32(4):273-278.

8. Palmisani M, Gasbarrini A, Brodano GB, et al. Minimally invasive percutaneous fixation in the treatment of thoracic and lumbar spine fractures. Eur Spine J. 2009;18(suppl 1):71-74. doi: 10.1007/s00586-009-0989-6.

9. Ni WF, Huang YX, Chi YL, et al. Percutaneous pedicle screw fixation for neurologic intact thoracolumbar burst fractures. J Spinal Disord Tech. 2010;23(8):530-537. doi: 10. 1097/BSD.0b013e3181c72d4c.

10. Kim DY, Lee SH, Chung SK, et al. Comparison of multifidus muscle atrophy and trunk extension muscle strength: percutaneous versus open pedicle screw fixation. Spine. 2005;30(1):123-129.

11. Bliemel C, Lefering R, Buecking B, et al. Early or delayed stabilization in severely injured patients with spinal fractures? Current surgical objectivity according to the Trauma Registry of DGU: treatment of spine injuries in polytrauma patients. J Trauma Acute Care Surg. 2014;76(2):366-373. doi: 10.1097/TA.0b013e3182aafd7a.

12. Vaccaro AR, Oner C, Kepler CK, et al. AOSpine Spinal Cord Injury \& Trauma Knowledge Forum. AOSpine thoracolumbar spine injury classification system: fracture description, neurological status, and key modifiers. Spine (Phila Pa 1976). 2013;38(23):2028-2037. doi: 10.1097/BRS.0b013e3182a8a381.

13. Brooks R, Rabin R, de Charro F (2003). The Measurement and Valuation of Health Status Using EQ-5D: A European Perspective. Dordrecht, The Netherlands: Kluwer Academic Publishers; 2003. 
14. Lamers LM, Stalmeier PF, McDonnell J, et al. Measuring the quality of life in economic evaluations: the Dutch EQ-5D tariff. Ned Tijdschr Geneeskd. 2005;149(28):1574-1578.

15. R Development Core Team. $R$ : A Language and Environment for Statistical Computing. Vienna, Austria: R Foundation for Statistical Computing; 2012.

16. Fox J, Weisberg S. An $\{R\}$ Companion to Applied Regression. 2nd ed. Thousand Oaks CA: SAGE Publications; 2011.

17. Cimatti M, Stefano F, Filippo P, et al. Pure percutaneous pedicle screw fixation without arthrodesis of 32 thoracolumbar fractures: clinical and radiological outcome with 36month follow-up. Eur Spine J. 2013;22(Suppl 6):925-932.

18. Schmidt OI, Strasser S, Kaufmann V, et al. Role of early minimal-invasive spine fixation in acute thoracic and lumbar spine trauma. Indian J Orthop. 2007;41(4):374-380. doi: 10. 4103/0019-5413.37003.

19. Van Dongen JM, van den Berg B, Bekkering GE, et al. Patient versus general population health state valuations: a case study of non-specific low back pain. Qual Life Res. 2017;26(6):1627-1633. doi: 10.1007/s11136-017-1497-5.

20. Stahel PF, VanderHeiden T, Flierl MA, et al. The impact of a standardized "spine damage-control" protocol for unstable thoracic and lumbar spine fractures in severely injured patients: a prospective cohort study. J Trauma Acute Care Surg. 2012;74(2):590-596. doi: 10.1097/TA.0b013e31827d6054.
21. Dimar JR, Carreon LY, Riina J, et al. Early versus late stabilization of the spine in the polytrauma patient. Spine. 2010;35(21):S187-192. doi: 10.1097/BRS.0b013e3181f32bcd.

22. Oner C, Shanmuganathan R, Jens RC. Spine traumawhat are the current controversies. J Orthop Trauma. 2017;31(4):S1-S6. doi: 10.1097/BOT.0000000000000950.

Disclosures and COI: No funds or grants were received for this manuscript. The authors declare no conflict of interest.

Corresponding Author: I. Sanli, MD, Department of Orthopaedic Surgery, Maastricht University Medical Centre+, P. Debyelaan 25 PO Box 58006202 AZ Maastricht, The Netherlands. Phone: +31-43-387 69 00; Email: ilknursanli@hotmail.com.

Published 31 December 2019

This manuscript is generously published free of charge by ISASS, the International Society for the Advancement of Spine Surgery. Copyright $\odot 2019$ ISASS. To see more or order reprints or permissions, see http://ijssurgery.com. 